\title{
Simple Composite Dipole Model for the Optical Modes of Strongly- Coupled Plasmonic Nanoparticle Aggregates
}

\author{
Richard W. Taylor, ${ }^{\dagger}$ Rubén Esteban, ${ }^{\ddagger}$ Sumeet Mahajan, ${ }^{\dagger}$ Roger Coulston, ${ }^{\S}$ Oren A. Scherman, ${ }^{\S}$ \\ Javier Aizpurua, ${ }^{\ddagger}$ and Jeremy J. Baumberg ${ }^{*} \dagger$ \\ ${ }^{\dagger}$ NanoPhotonics Centre, Cavendish Laboratory, University of Cambridge, CB3 0HE, U.K. \\ ${ }^{\ddagger}$ Centro de Fisica de Materiales, Centro Mixto CSIC-UPV/EHU and Donostia International Physics Center (DIPC), Donostia-San \\ Sebastián, 20018, Spain \\ ${ }^{\S}$ Melville Laboratory for Polymer Synthesis, Department of Chemistry, University of Cambridge, Cambridge, CB2 1EW, U.K.
}

ABSTRACT: Self-assembled strongly coupled plasmonic aggregates exhibit optical spectra which show complex plasmonic resonances. To understand the optics of such systems, we introduce an effective composite dipole model extending previous effective models of aggregates into the plasmonic domain. The ingredients in this model are found by comparing the time-resolved extinction of self-assembling growing aggregates of gold nanoparticles spaced by rigid sub-nm gaps to recent rigorous electromagnetic simulations of this geometry. The highly reproducible spectral signatures from experiments match our simulations, confirming that the electromagnetic response of such fractal plasmonic clusters can be well-understood in terms of embedded straight chains of plasmonically coupled nanoparticles surrounded by an optically decoupled halo of dimers. We show how to derive simple analytical formulas that lead to rapid extraction of key parameters from such

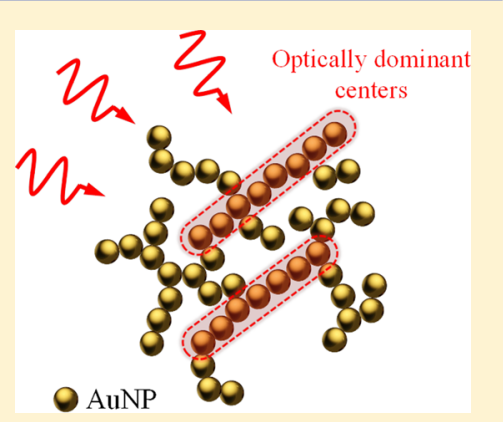
experimental spectra and which properly account for the long-wavelength lineshapes. In particular, we find these effective parameters describe the extent of plasmon delocalization along such chains, the eccentricity of these optically dominant cores, and the fraction of nanoparticles active within them. This underpins applications which depend on spectral selectivity and field enhancements in such tightly coupled plasmonic systems.

\section{INTRODUCTION}

Self-assembled networks of plasmonic nanoparticles present a versatile and highly functional class of nanomaterial. The origin of the strong optical response in plasmonic nanoparticle systems stems from a resonant coupling between the oscillating electrons in a metal and light at optical frequencies. ${ }^{1-6}$ The remarkable ability to "capture" and tightly confine light down to subnanoscopic dimensions has been seized upon in a wide range of interdisciplinary applications. Surface-enhanced Raman scattering (SERS), for example, is a well-known beneficiary of aggregate plasmonics. ${ }^{7-13}$ Coagulates of nanoparticles promote extremely large optical enhancements at the nanoscale, ${ }^{14-19}$ enabling even single-molecule detection within the gaps of closely coupled nanoparticles. ${ }^{20-23}$ Furthermore, plasmonic aggregates have also demonstrated potential in various biomedical applications including in vivo therapeutics such as photothermal cancer therapy, ${ }^{24,25}$ via light-induced resonant plasmonic heating, ${ }^{26,27}$ in bioassays for drug testing, ${ }^{28}$ and also intracellular imaging of biomolecules. ${ }^{29-31}$ Moreover, while individual nanoparticles are currently suggested to enhance solar cell performance, ${ }^{32-34}$ nanoparticle aggregates may offer an alternative route.

Despite such wide-ranging applicability to a variety of disciplines, elucidation as to precisely where in the aggregate the routinely harnessed strong optical resonances are supported has been somewhat limited. ${ }^{15,35-40}$ The challenges can in part be attributed to irreproducible and inconsistent interparticle spacings within experimentally fabricated coagulates and to the naturally complicated topology of the aggregate itself. When assemblies of noble metal nanoparticles are spaced much closer than their diameters, the localized plasmons in each gap can be strongly coupled to each other. ${ }^{41}$ The strength and character of this coupling is highly sensitive to the particle separation and is crucial for the optical response of the composite structure. ${ }^{42-44}$ Furthermore, determining the nature of the extended electromagnetic states resulting from this strong coupling is further complicated by the essentially random, quasi-fractal geometry of the aggregate. ${ }^{44-46}$

In this paper, using nanoaggregates that possess fixed and rigid sub-nm nanoparticle spacing, we are able to closely compare experiments with spectra produced from full electromagnetic simulations as well as with a simple effective model of great utility. We confirm the view that embedded chain structures within each aggregate define the far-field spectral response. In particular, the longitudinal plasmon mode of the longest embedded chains inside the aggregates accounts for both the long wavelength peak and the spectral line shape observed. From this perspective, we can thus extend existing effective optical models used to describe composites of dielectric nanoparticles, such as carbon, ${ }^{47}$ into the plasmonic

Received: September 10, 2012

Revised: October 30, 2012

Published: November 2, 2012 
(a)
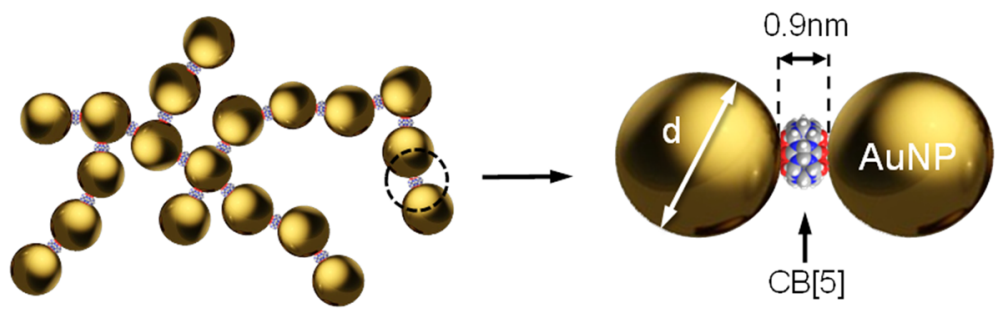

(b)

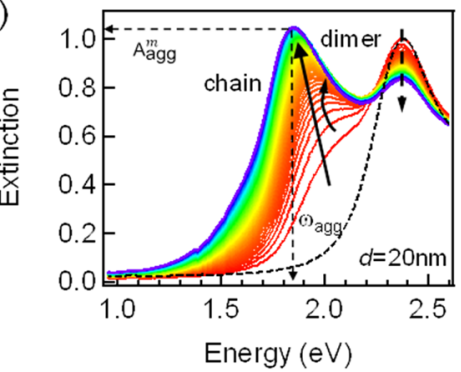

(d)

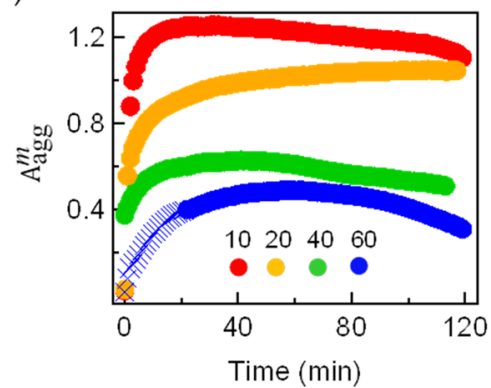

(c)

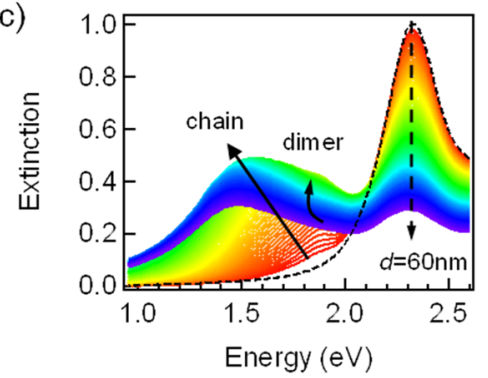

(e)

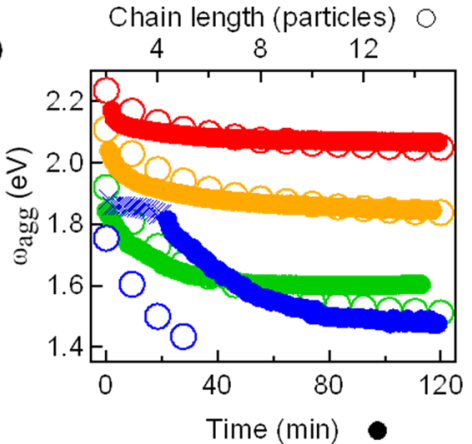

Figure 1. (a) Schematic of a quasi-fractal aggregate of AuNPs with CB[5] spacers. (right) Close view of an AuNP dimer separated by $0.9 \mathrm{~nm}$. (b and c) Time-resolved extinction spectra for an aggregating AuNP:CB[5] solution with $d=(\mathrm{b}) 20$ and (c) $60 \mathrm{~nm}$. Spectra separated by $60 \mathrm{~s}$; arrows guide the eye. The dimer and chain modes of the aggregate are highlighted. Normalized time-resolved peak extinction $(\mathrm{d})$ and aggregate peak resonance energy $(\mathrm{e}, \mathbf{0})$ of the low energy (chain) mode as a function of AuNP diameter (nm). The initial response for $60 \mathrm{~nm}$ AuNPs is that of a dimer-like mode. The simulated extinction peak of straight AuNP chains with $0.9 \mathrm{~nm}$ interparticle gaps also shown (e, $O$ ) vs chain length (top axis).

domain in order to account for the dominant chain substructures. The resulting composite dipole model (CDM) for plasmonic aggregates fully describes the dominant lowenergy components of the experimental spectral line shape, is simple enough to use to track real-time aggregation and provides estimates for the underlying chain morphology based only on the spectral extinction. The insights afforded by the CDM should enable greater understanding of the optical properties of plasmonic aggregates. We emphasize that the presence of well-defined and subnanometer particle separations, which introduce strong particle interactions and chain-like modes, is critical for this effective composite dipole model which can only then account for the long wavelength optical response of the whole aggregate. This simplified description remains valid in spite of the presence of more complex interparticle interactions within the cluster.

\section{EXPERIMENTAL: FABRICATION AND SPECTROSCOPY}

Self-assembled nanoaggregates are formed here through the aggregation of monodisperse Au nanoparticles (AuNP) in the presence of a rigid macrocyclic "glue" molecule known as cucurbit $[5]$ uril $(\mathrm{CB}[5])$. The $\mathrm{CB}[5]$ is known to bind metallic colloids together, reproducibly fixing the interparticle separation, $l_{\text {gap }}$, at precisely $0.9 \mathrm{~nm}^{48-50}$ This control over the particle separation not only ensures that the nanoaggregates exhibit strong optical resonances but also ensures reproducible optical modes. The general topology of the aggregates considered in this work, shown schematically in Figure 1a, is quasi-fractal, consistent with known diffusion-limited colloid aggregation (DLCA) kinetics, ${ }^{44-46}$ as evidenced in transmission electron microscopy. ${ }^{49}$

During aggregate growth, time-resolved millisecond visible and near-infrared (NIR) spectroscopy is used to track assembly of monomers of nominal diameters $d=10-60 \mathrm{~nm}$ in solution, as shown in Figure $1 \mathrm{~b}$ and $c$. Initially the single particle plasmon resonance around $520 \mathrm{~nm}$ is observed. Aggregation of plasmonic nanoparticles produces additional lower-energy optical resonances, owing to the strong electromagnetic nearfield coupling between closely packed nanoparticles. As we show below, the spectral extinction displays at least two separate resonant contributions for wavelengths beyond the single particle mode, originating from optical modes associated with dimers and with long chains. ${ }^{38,49}$ The lower-energy mode from the long chains grows stronger in intensity with aggregation time. In this work, we refer to the spectral extinction of this mode as $A_{\text {agg }}$ occurring at a peak energy $\omega_{\text {agg }}$. Throughout, we also subtract off the residual single-particle plasmon spectral component to better concentrate on the key spectral features that emerge during growth. ${ }^{49}$ The maximum value of extinction for the aggregates, $A_{\mathrm{agg}}^{\mathrm{m}}$ is extracted in time during the assembly process and shown in Figure 1d. For all 


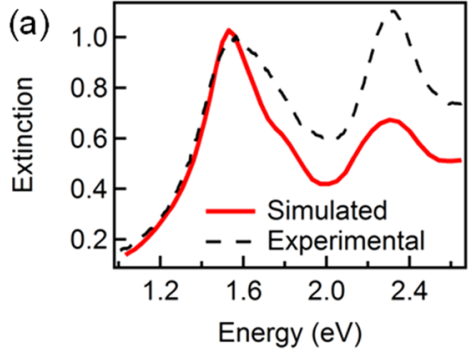

(c)

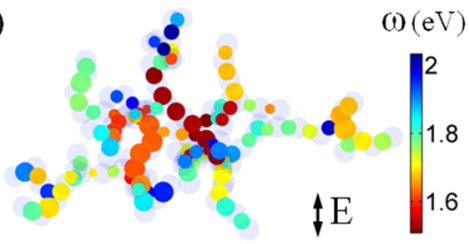

(b)

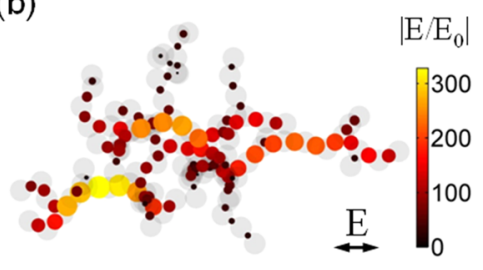

(d)

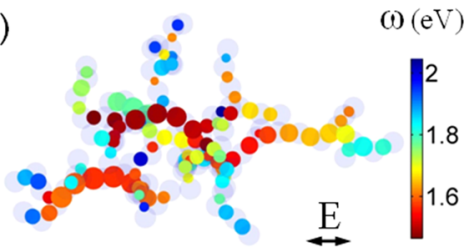

Figure 2. Simulated 3D 100-sphere aggregate of similar structure to those created experimentally $\left(d=40 \mathrm{~nm}, l_{\text {gap }}=0.9 \mathrm{~nm}\right)$. (a) Calculated extinction cross-section of the aggregate (red solid) compared to experimental spectrum (black dashed). (b-d) Spatial field distribution of the modes (polarization of incident plane wave marked). The gray spheres show the particles forming the aggregates, while the solid spheres give the field information at the gap positions. The 3D aggregates have been projected onto the plane perpendicular to the propagation vector. In $b$ both the color and point area denote the strength of the fields at the spectral position corresponding to $\omega_{\text {agg. }}$ In $\mathrm{c}$ and $\mathrm{d}$, the point color denotes the photon energy of maximum field enhancement at each gap, and the point area gives the field enhancement at this energy.

diameters studied, the growth of the peak mode is initially fast, followed by saturation. The concomitant evolution in the resonance energy of the mode, $\omega_{\text {agg }}$, is similarly tracked in time (O, Figure 1e). The growth kinetics reveals a similar trend in both the saturation of the extinction maxima and also in the redshift of the peak energy. Thus, it is clear that $A_{\mathrm{agg}}^{\mathrm{m}}$ and $\omega_{\mathrm{agg}}$ originate from the same optical mode supported within the aggregate, with the same structural origin, which we later identify to be constituent chains.

While the emergence and evolution of lower-energy optical resonances upon aggregation of plasmonic nanoparticles is well-known, and the observed extinction is consistent with previous reports, ${ }^{51-57}$ the precise structural origin of modes has been unclear until recently. Previous work to explain the universal features of the optical spectra based upon the general structure of aggregates have yielded mostly qualitative agreement between theory and experiment. Nonetheless, it is found that strong red-shifting of the aggregate mode is observed for increasing nanoparticle size, number, and packing density within aggregates possessing both quasi-fractal and crystal lattice morphologies. ${ }^{52,53,58,59}$ These results for coagulated particles are understood to be the consequence of electromagnetic coupling between the induced dipole modes of particles spaced less than a diameter apart. It is thus not surprising that similar optical behavior is observed in experimental dimers and linear chains ${ }^{40,42,60-64}$ and is also seen in similar particle-particle electrodynamic calculations on nanopairs and chain assemblies. ${ }^{55,65,66}$ While the coinciding resonance energies of straight nanochains and compositionally similar aggregates have been noted previously, ${ }^{38,49,55}$ only recently did we discuss how these apparent chain modes are both supported and distributed within the large fractal motif of large three-dimensional (3D) aggregates. ${ }^{67}$ Hence, we first discuss this explanation before using it to develop a simple effective composite dipole model for the aggregation process.

Theoretical Simulations. To provide an initial description, we first match our spectral shifts with full electrodynamic calculations of the optical response for 1-dimensional (1D) chains. ${ }^{67,68}$ The model structures are composed of an increasing number of longitudinally coupled AuNPs with the same particle separation as fabricated experimentally. The nanoparticles modeled use $\mathrm{Au}$ dielectric values from Johnson and Christy with size corrections. ${ }^{69}$ The low energy peak modes for the straight 1D chains of different length and particle diameter are displayed ( $O$, Figure 1e) together with $\omega_{\text {agg }}$ from the experimental aggregates $(\mathbf{O}$, Figure 1e). Good correspondence between the mode energies of the evolving experimental aggregate peak and that of the simulated 1D chain is apparent. For the larger $d=60 \mathrm{~nm}$ nanoparticles, it appears that the aggregates grown are composed of far fewer particles than those observed for the aggregates of smaller particles (likely due to a different surface binding rate), and thus, a correlation with smaller effective $1 \mathrm{D}$ chain lengths is anticipated.

To further explore the correlation between the aggregate peak energy and those of $1 \mathrm{D}$ chains, we summarize our recently published simulations of the optical response for an arbitrary 3D aggregate, ${ }^{67,70}$ formed from 100 AuNPs of $40 \mathrm{~nm}$ diameter with a separation of $0.9 \mathrm{~nm}$, exhibiting DLCA-like topology (Figure 2). A more detailed discussion on the calculation of the optical response from these aggregates has been reported by Esteban et al. ${ }^{67}$ Remarkably, the spectral extinction matches that measured experimentally from a broad ensemble average (Figure 2a). Interestingly this result suggests that precise knowledge of the arrangement of nanoparticles within these fixed-gap aggregates is not important, but rather some other feature robustly determines the optical response.

We identify embedded chain structures as being responsible for the optical behavior observed. The near field enhancement $\left(E / E_{0}\right)$ within the particle gaps that support the low energy mode are observed to be especially strong for $1 \mathrm{D}$ chains oriented roughly parallel to the optical polarization (Figure $2 b){ }^{35,40}$ The distribution of the resonant modes in this aggregate for two different polarizations further illustrates that the low energy modes are supported by $1 \mathrm{D}$ chains that extend in a direction selected by the polarization (Figure $2 c$ and $d$ ). These chain modes mimic those of straight chains and are remarkably robust against disorder. ${ }^{67,71-74}$ These local nearfield distributions within the simulated aggregate thus 
(a)

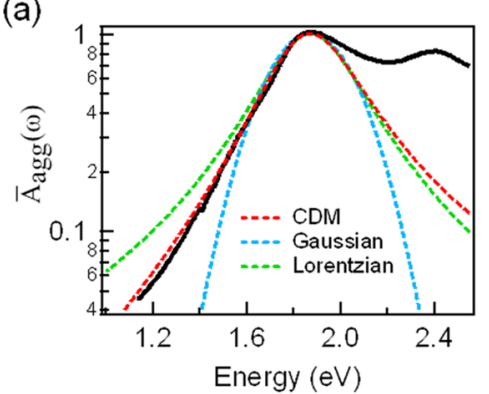

(b)

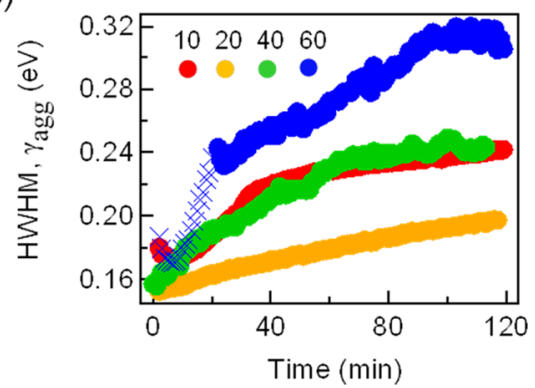

Figure 3. (a) Experimental spectrum (solid, $120 \mathrm{~min}, d=20 \mathrm{~nm}$ ) with CDM fit from eq 3, together with Gaussian and Lorentzian fits of same halfwidth. (b) Fitted HWHM $\left(\gamma_{\text {agg }}\right)$ from the experimental extinction from eq 4.

demonstrate that the lowest energy mode is fundamentally supported by embedded, weakly interacting chains within the aggregate. ${ }^{75}$ These optically functional chains are selected by the incident light and scale in length with the overall size of the grown aggregate, up to the limit that coupled plasmons may delocalize along a chain. ${ }^{76,77}$ By this we mean that, instead of individual decoupled gap resonances (i.e., dimer modes), many gaps between particles along the kinked linear chain are coupled together. Moreover, it is the optical mode of these chains specifically that has been previously attributed to the full aggregate structures.

In addition to the presence of long chains within the aggregate, both dimers and single particle-like modes are also seen to be optically supported, and it is these that account for the higher energy modes observed within the aggregate. In addressing how these three modes can be simultaneously supported within a typical aggregate, Figure $2 \mathrm{c}$ and $\mathrm{d}$ reveals the dominant long chain mode to be centrally embedded within a "halo" of surrounding dimers and single particles. Both experiments and theory thus point to this unexpected result: a random DLCA plasmonic aggregate with fixed nanogaps selfseparates into different optically dominant domains that are spatially separated from each other.

The low-energy embedded chain resonances thus seen in the far-field also account for the near-field properties of such aggregates with the strongest field enhancements occurring within the interparticle gaps of these chains. Previous studies on quasi-fractal aggregates, ${ }^{14,16,78,79}$ where the gap spacing is highly variable, show that regions of the near-field landscape are governed by points of high local anisotropy which yield the strongest enhancements. ${ }^{14,17,80}$ Here, the near-field profile observed for the simulated aggregate is more consistent with the field localization found for nanopairs and linear chain structures, ${ }^{27,35,81-85}$ supporting the embedded chain perspective. The near-field properties of the aggregate, such as those exploited for SERS, can thus also be understood from the embedded chain perspective. ${ }^{86}$

Effective Optical Model. Knowing that embedded chains account for the near- and far-field optical functionality of an aggregate, we now formulate a simple optical model to report parameters related to these structures during real-time growth. This is extremely desirable since full simulations are extremely resource intensive, and this problem is further exacerbated by the large ensembles of aggregates often used. One approach suited for weakly interacting clusters is to depict the optical response via an effective dielectric function. ${ }^{87}$ Alternatively, existing exact numerical methods that implement and solve for the particle-particle interactions, after Mie, for arbitrary plasmonic aggregates, also require the topology of interest to be explicitly defined. ${ }^{19,51,66,88}$ However, the precise $3 \mathrm{D}$ topology of an aggregate in situ is almost always unknown. Results from the previous section however indicate that in strongly coupled fixed-gap plasmonic aggregates, the characteristics of the dominant lower energy resonance may be robustly inferred in terms of chain modes through the spectral extinction alone, and thus free from the requirement to know the precise details of nanoparticle arrangement.

We introduce here an effective dipole description for the optically dominant chain kernel(s) within an aggregate of strongly interacting particles. To do so, this composite dipole model uses a simple and compact expression, similar to the quasistatic dipolar resonance of a spheroid, to track the spectral extinction. From this assumption, the extinction of the aggregate, $A_{\text {agg, }}$ can then be expressed as ${ }^{4,63}$

$$
A_{\mathrm{agg}}=\frac{V_{\mathrm{agg}}}{c} \omega \operatorname{Im}\left\{\frac{\varepsilon_{\mathrm{Au}}-\varepsilon_{\mathrm{m}}}{\varepsilon_{\mathrm{m}}+L_{\mathrm{agg}}\left(\varepsilon_{\mathrm{Au}}-\varepsilon_{\mathrm{m}}\right)}\right\}
$$

where $V_{\text {agg }}$ refers to the volume of the active plasmonic system, $\omega$ is the angular frequency, $\varepsilon_{\mathrm{m}}$ is the dielectric constant of the embedding medium, $\varepsilon_{\mathrm{Au}}$ is the dielectric function of gold, $c$ is the speed of light in the medium, $L_{\text {agg }}$ the depolarisation factor, and $\operatorname{Im}\{\}$ is the imaginary part. Substituting into eq 1 the Drude model, $\varepsilon_{\mathrm{Au}}=\varepsilon_{\infty}-\omega_{\mathrm{p}}^{2} / \omega(\omega+i \Gamma)$ for gold, where $\varepsilon_{\infty}$ gives the screening from the d-electrons, $\omega_{\mathrm{p}}$ is the plasma frequency, and $\Gamma$ describes the material damping, and taking the imaginary part gives exactly

$$
A_{\mathrm{agg}}=\frac{a_{\mathrm{agg}} V_{\mathrm{agg}}}{\mathrm{c}} \frac{\omega^{2}}{\left(\omega^{2}-v_{\mathrm{agg}}{ }^{2}\right)^{2}+\delta_{\mathrm{agg}}{ }^{4}}
$$

where

$$
\begin{aligned}
& {v_{\mathrm{agg}}}^{2}=\omega_{\mathrm{p}}{ }^{2} / \bar{\varepsilon}-\Gamma_{\mathrm{agg}}{ }^{2} / 2 \\
& \delta_{\mathrm{agg}}{ }^{4}=\Gamma_{\mathrm{agg}}{ }^{2}\left(v_{\mathrm{agg}}{ }^{2}+\Gamma_{\mathrm{agg}}{ }^{2} / 4\right) \\
& a_{\mathrm{agg}}=\Gamma_{\mathrm{agg}} \omega_{\mathrm{p}}{ }^{2} \varepsilon_{\mathrm{m}}\left[\bar{\varepsilon} L_{\mathrm{agg}}\right]^{-2} \\
& \bar{\varepsilon}=\varepsilon_{\infty}+\left(L_{\mathrm{agg}}{ }^{-1}-1\right) \varepsilon_{\mathrm{m}}
\end{aligned}
$$

In this model we assume the aggregate damping term $\Gamma_{\text {agg }}=\Gamma$ to phenomenologically include the different broadening mechanisms within the experimental aggregates. We consider that $V_{\text {agg, }} L_{\text {agg }}$ and also $\Gamma_{\text {agg }}$ are parameters to be fitted to correctly account for the optical properties of the aggregates. The aggregate peak energy is found at $\omega_{\text {agg }}=v_{\text {agg }}\left(1+e_{\text {agg }}\right)^{1 / 4}$ 
where $e_{\text {agg }}=\bar{\Gamma}_{\text {agg }}{ }^{2}\left(1+\bar{\Gamma}_{\text {agg }}{ }^{2} / 4\right)=\delta_{\text {agg }}{ }^{4} / v_{\text {agg }}{ }^{4}$ and $\bar{\Gamma}_{\text {agg }}=\Gamma_{\text {agg }} / v_{\text {agg }}$ $=\sqrt{ } 2\left[\left(1+e_{\mathrm{agg}}\right)^{1 / 2}-1\right]^{1 / 2}$. The maximum extinction of the aggregate is then $A_{\mathrm{agg}}^{\mathrm{m}}=a_{\mathrm{agg}} V_{\mathrm{agg}}\left[c \Gamma_{\mathrm{agg}}^{2}\right]^{-1}$. Normalizing the spectral extinction to the peak, we thus obtain a model for the normalized aggregate extinction supported by embedded dipole resonances,

$$
\bar{A}_{\mathrm{agg}}=\frac{A_{\mathrm{agg}}}{A_{\mathrm{agg}}^{\mathrm{m}}}=\frac{\beta_{\mathrm{agg}} \bar{\omega}^{2}}{1+e_{\mathrm{agg}}^{-1}\left[1-\bar{\omega}^{2}\right]^{2}}
$$

where $\bar{\omega}=\omega / v_{\text {agg }}$ and $\beta_{\text {agg }}=\bar{\Gamma}_{\text {agg }}^{2} / e_{\text {agg }}$. One immediate result from eq 3 is that the CDM naturally reproduces the low energy "tail" line shape always observed for the aggregate mode, shown in Figure 3a. ${ }^{55,89}$ In contrast, fits of this tail using Gaussian or Lorentzian resonant shapes fail. This suggests that the electric dipole mode of the embedded chain structures is a natural description of the strongest contribution to the aggregate optics. We emphasize that we obtain a good fit to the experimental results thanks to the simple quasistatic model, introduced in eq 1 , because our approach includes the complex interparticle couplings that give rise to multipolar terms, as well as retardation in the interactions that play a role for extended clusters or large particles. Both effects are included by means of the right choice of parameters in eq 2 , and the obtained results are interpreted with input from the exact electrodynamical calculations. We find the fit of eq 3 works for all the different diameters considered and, throughout the assembly process, to better than $5 \%$.

The fit of eq 3 via $e_{\text {agg }}$ to experimental extinction allows for the extraction of the aggregate peak half-width at half-maximum (HWHM, $\gamma_{\text {agg }}$ ) on the low energy side,

$$
\gamma_{\mathrm{agg}}=\omega_{\mathrm{agg}}\left[1-\left(s-\sqrt{s^{2}-1}\right)^{1 / 2}\right]
$$

where we define for convenience $s=2-\left[1+e_{\mathrm{agg}}\right]^{-1 / 2}$. This also means that from a simple measurement of the experimental HWHM it is possible to directly extract the fit parameter, $e_{\text {agg. }}$. The evolution of the fitted HWHM (Figure $3 \mathrm{~b}$ ) during aggregation reveals a persistently increasing broadening with time. This can be understood to be a result of increased radiative damping from the growing embedded long chains in addition to inhomogeneous broadening and other losses. ${ }^{90}$

In addition to the damping, the CDM also permits the extraction of the evolving depolarisation factor $L_{\mathrm{agg}}$ that describes the effective eccentricity of the composite mode, Figure $4 \mathrm{a} .{ }^{91} L_{\mathrm{agg}}$ is initially equal to that of an individual AuNP sphere: $L_{\mathrm{sp}}=0.23$ (while $L_{\mathrm{sp}}=1 / 3$ for a small perfect Drude metal sphere) but with subsequent growth of the aggregate and thus the constituent chains, $L_{\text {agg }}$ drops to an order of $\approx 0.1$ giving effective ellipsoidal eccentricities of up to $90 \%$. This interpretation of the growth in time is broadly consistent with the expectation of the effective dipole mode being required to "extend" over a longer spatial volume as the effective embedded chain length grows within the expanding aggregate.

Owing to the agreement between theory and experiment for describing the low energy line shape, we extract a parameter that can be understood as an effective particle number, $N_{p}$, in each dominant low energy chain, by matching the experimental $\omega_{\text {agg }}$ with the equivalent 1D simulation, shown in Figure $4 \mathrm{~b}$. A similar extraction on the simulated aggregate of Figure $2 \mathrm{~b}$, for which we determine $N_{\mathrm{p}} \sim 12$, supports the suggestion that $N_{\mathrm{p}}$ is directly related to the length of the low-energy optically dominant chain. Typically the observed $N_{\mathrm{p}}$ will be limited by
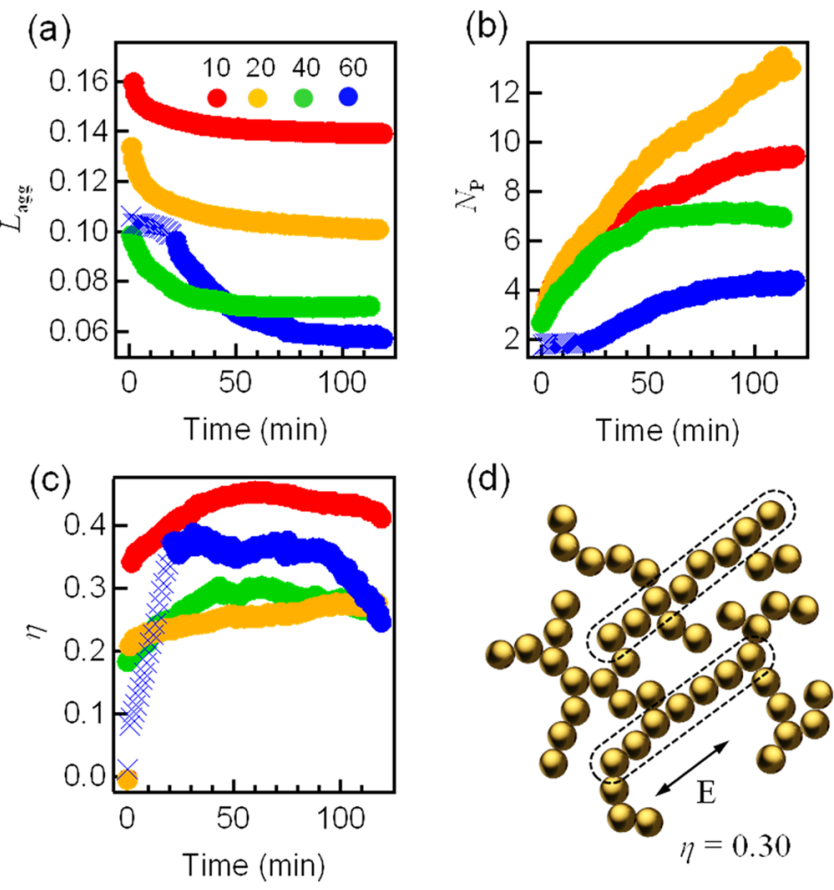

Figure 4. Time evolution, as a function of diameter $d$, for different parameters characterizing the geometry of the growing experimental clusters. (a) Extracted effective depolarisation factor $L_{\text {agg }}$. (b) Interpolated effective number of particles in the chains $N_{\mathrm{p}}$ obtained from $\omega_{\text {agg }}$ (c) Experimentally extracted effective fraction of optically dominant NPs composing functional chains $\eta$. (d) Schematic illustration of optically dominant chains within an arbitrary aggregate of 50 particles with $\eta=0.30$.

the extent to which the plasmon can delocalize down the strongly coupled chain.

Finally, insight into the actual proportion of NPs that contribute to the dominant low-energy optical response within the aggregate is achieved by looking at the absolute strength of the spectra. To do this we recast eq 2 to examine the role of $V_{\text {agg }}$ so as to explicitly account for the resonant chain structure. This is achieved by comparing the effective optical volume $V_{\text {agg }}$ of the aggregate in eq 1 to the physical volume of the particles supporting the plasmonic resonances. ${ }^{4,63}$ For small spheres and other simple situations, $V_{\text {agg }}$ equals the volume of the plasmonic system. For the case of straight chains, $V_{\text {agg }}$ has also been compared to the physical size of the resonant structure. ${ }^{4,63} \mathrm{We}$ now consider $V_{\text {agg }}$ for an optically active aggregate such as that in Figure 2. We model each aggregate as being composed of $n_{\mathrm{sp}}$ particles, of which a fraction belongs to $n_{\mathrm{ch}}$ active core chains where each chain is composed of $N_{\mathrm{p}}$ particles of diameter $d$. The physical volume of the active chains $V_{\mathrm{agg}}^{\prime}=n_{\mathrm{ch}} N_{\mathrm{p}} \pi d^{3} / 6$, may then be related to the effective optically functional volume $V_{\text {agg }}$ through $V_{\text {agg }}=g\left(N_{\mathrm{p}}\right) V_{\text {agg }}^{\prime}$. The factor $g\left(N_{\mathrm{p}}\right)$ accounts for effects such as scattering contributions and retardation that make $V_{\text {agg }}$ different from the actual physical volume of the chains $\left(V_{\text {agg }}^{\prime}\right)$. As $g\left(N_{\mathrm{p}}\right)$ is calculated using rigorous modeling, it allows for introducing the effects of retardation in the interactions along each chain as well as the multipolar contribution to the particle-particle interactions. We can use our simulations of straight chains of length $N_{\mathrm{p}}$ to calibrate $g\left(N_{\mathrm{p}}\right)$, where this calibration depends implicitly on the particle diameter, interparticle gap, and the material. The extinction maximum of the nanoaggregate can then be expressed as 


$$
A_{\mathrm{agg}}^{\mathrm{m}}=\frac{\pi d^{3} n_{\mathrm{ch}} N_{\mathrm{p}} g\left(N_{\mathrm{p}}\right)}{6 c f_{\mathrm{agg}}}
$$

where $f_{\mathrm{agg}}=\Gamma_{\mathrm{agg}}{ }^{2} \mathrm{a}_{\mathrm{agg}}{ }^{-1}$. In our experiments, we can normalize $A_{\text {agg }}^{\mathrm{m}}$ by the equivalent extinction produced by the single NPs in the initial unaggregated state, $A_{\mathrm{sp}}^{\mathrm{m}}$ (isolated spheres, such that $N_{\mathrm{p}}=1$ ), which is proportional to the initial number of single particles, $n_{\mathrm{sp}}$ that will form the aggregates. It is then possible to define an effective proportion, $\eta$, of particles responsible for the dominant low-energy response as

$$
\eta=\frac{N_{\mathrm{p}} n_{\mathrm{ch}}}{n_{\mathrm{sp}}}=\frac{g(1)}{g\left(N_{\mathrm{p}}\right)} \frac{A_{\mathrm{agg}}^{\mathrm{m}}}{A_{\mathrm{sp}}^{\mathrm{m}}} \frac{f_{\mathrm{agg}}}{f_{\mathrm{sp}}}
$$

All the necessary terms in this expression can be obtained from the straight chain simulations and from the experimental measurements of the extinction peak.

To understand the physical meaning of the obtained parameter $\eta$, it is useful to consider a simple aggregate constituted by both optically inactive particles and resonant $1 \mathrm{D}$ chains of fixed length $N_{\mathrm{p}}$, the latter parallel to the incident polarization and not interacting with each other. Here $\eta$ corresponds to the proportion of the particles in this simple aggregate that belong to an active chain in order to produce the same maximum peak $A_{\mathrm{agg}}^{\mathrm{m}}$ as for the experimental aggregate under consideration. We thus identify $\eta$ as the effective proportion of active particles that contribute to the lowest energy peak: $\eta$ gives information on how many particles belong to $1 \mathrm{D}$ chains inside the aggregates that are responsible for the resonant extinction. We note that, for the complex clusters realized in experiments, our aim is not to yield exact quantitative values but rather to understand the general trends.

Figure $4 c$ reveals that in all the cases considered the effective NP fraction involved in chains increases consistently through the aggregation process and saturates at around $\sim 30 \%$, shown schematically in Figure 4d. From this we may conclude that only a moderate fraction of the aggregated NPs actually support the optically dominant chains at a given instance. This observation is indeed supported by the full simulations of Figure 2 and also suggested experimentally. ${ }^{92,93}$

\section{SUMMARY}

The experimentally observed spectral extinction of aggregates is very well correlated with chain modes seen in $1 \mathrm{D}$ and $3 \mathrm{D}$ simulations. We interpret this to imply that the optically functional constituent structures within the structurally complex aggregates are actually just a few almost-linear optically selected chains embedded within a halo of surrounding dimers. The simple optical modes of the chains are remarkably independent of disorder as long as the gap separation is fixed. ${ }^{67}$ This independent embedded-chain model explains the evolving extinction seen in Figure 1 in both mode energy and extinction intensity, as the chains grow inside the aggregates. From this perspective of active chains, an effective CDM can be successfully applied to depict the dominant optical mode of the chains so as to reproduce the low energy spectral extinction and track the progress of the growing chains. The success of the CDM is remarkable given the assumptions of the quasi-static approximation while omitting radiative damping in our starting eq 1 , averaging over many quasi-fractal topologies, and assuming straight chains. The likely robustness of the model stems from the features of the dominant electric dipole mode of a strongly coupled chain, which are surprisingly insensitive to disorder, ${ }^{87,74,94,95}$ and which support only limited delocalization of plasmon coupling along the chains. The chain model presented should thus enable more insightful use of plasmonic nanoaggregates. $^{86}$

\section{CONCLUSION}

In conclusion, we assemble and model AuNP aggregates that possess well-defined plasmon resonances by virtue of the fixed and reproducible nanoparticle gaps defined by a rigid molecular spacer. Although the resulting nanostructures present multimodal optical resonances, a simple composite dipole model can successfully describe the long wavelength resonance and tail of the lowest energy peak and allow robust characterization of the real-time dynamics. Furthermore, the identification of the aggregate resonance with the excitation of the constituent chains allows us to define and track an effective mean number of particles $\left(N_{\mathrm{p}}\right)$ in these chains and an effective proportion of active particles $(\eta)$. This approach to understanding complex extinction spectra is very useful for applications that are sensitive to specific plasmons within aggregates, and more generally for a much broader range of optically active nanoparticle assemblies.

\section{AUTHOR INFORMATION}

\section{Corresponding Author}

*E-mail: jjb12@cam.ac.uk.

\section{Notes}

The authors declare no competing financial interest.

\section{ACKNOWLEDGMENTS}

This work was supported by EPSRC EP/F059396/1, EP/ G060649/1, and EU NanoSci-E+ CUBiHOLE grants , and projects FIS2012-19609-C02-01 and EUI200803816 from the Spanish Ministry of Science and Innovation, and ETORTEK project "nanoiker" from the Department of Industry of the Basque Government. We thank Alexander Kuhn for many helpful discussions.

\section{REFERENCES}

(1) Maier, S. A. Plasmonics - Fundamentals and Applications; Springer: New York, N.Y., 2005.

(2) Barnes, W. L.; Dereux, A.; Ebbesen, T. W. Nature 2003, 424, 824-830.

(3) Myroshnychenko, V.; Rodríguez-Fernández, J.; Pastoriza-Santos, I.; Funston, A. M.; Novo, C.; Mulvaney, P.; Liz-Marzán, L. M.; García de Abajo, F. J. Chem. Soc. Rev. 2008, 37, 1792-1805.

(4) Bohren, C. F.; Huffman, D. R. Absorption and Scattering of Light by Small Particles; John Wiley \& Sons, Inc: New York, N.Y., 1998.

(5) Van Duyne, R. P.; Haynes, C. L.; Haes, A. J.; Mcfarland, A. D. Top. Fluoresc. Spectrosc. 2005, 8, 47-99.

(6) Novotny, L.; Hecht, B. Principles of Nano-Optics, 1st ed.; Cambridge University Press: Cambridge, U.K., 2006.

(7) Ghosh, S. K.; Pal, T. Chem. Rev. 2007, 107, 4797-862.

(8) Faulds, K.; Littleford, R. E.; Graham, D.; Dent, G.; Smith, W. E. Anal. Chem. 2004, 76, 592-598.

(9) Moskovits, M. Rev. Mod. Phys. 1985, 57, 783-826.

(10) Otto, A.; Futamata, M. Surface-Enhanced Raman Scattering Physics and Applications; Kneipp, K., Moskovits, M., Kneipp, H., Eds.; Springer-Verlag: Berlin, Germany, 2006.

(11) Jin, R. Angew. Chem., Int. Ed. 2010, 49, 2826-2829.

(12) Kneipp, J.; Kneipp, H.; McLaughlin, M.; Brown, D.; Kneipp, K. Nano Lett. 2006, 6, 2225-2231. 
(13) Weitz, D. A.; Lin, M. Y.; Sandroff, C. J. Surf. Sci. 1985, 158, $147-164$.

(14) Karpov, S.; Gerasimov, V.; Isaev, I.; Markel, V. Phys. Rev. B 2005, 72, 205425-205433.

(15) Stockman, M. I. Phys. Rev. E 1997, 56, 6494-6507.

(16) Drachev, V. P.; Bragg, W. D.; Podolskiy, V. A.; Safonov, V. P.; Kim, W.; Ying, Z. C.; Armstrong, R. L.; Shalaev, V. M. J. Opt. Soc. Am. 2001, 18, 1896-1903.

(17) Stockman, M.; Pandey, L.; Muratov, L.; George, T. Phys. Rev. Lett. 1994, 72, 2486-2489.

(18) Shalaev, V. M. In Handbook of Nanostructured Materials and Nanotechnology; Nalwa, H. S., Ed.; Academic Press: New York, 2000.

(19) Shalaev, V. M. In Nonlinear Optics of Random Media; Shalaev, V. M., Ed.; Springer-Verlag: Berlin, Germany, 2000.

(20) Kneipp, K.; Wang, Y.; Kneipp, H.; Perelman, L. T.; Itzkan, I.; Dasari, R. R.; Feld, M. S. Phys. Rev. Lett. 1997, 78, 1667-1670.

(21) Xu, H.; Aizpurua, J.; Käll, M.; Apell, P. Phys. Rev. E 2000, 62, $4318-4324$.

(22) Campion, A.; Kambhampati, P. Chem. Soc. Rev. 1998, 27, 241250.

(23) Lim, D.; Jeon, K.; Kim, H. M.; Nam, J.; Suh, Y. D. Nat. Mater. 2010, 9, 60-67.

(24) El-Sayed, I. H.; Huang, X.; El-Sayed, M. Cancer Lett. 2006, 239, 129-135.

(25) Lal, S.; Clare, S. E.; Halas, N. J. Acc. Chem. Res. 2008, 41, 18421851.

(26) Steiner, M.; Debus, C.; Failla, A. V.; Meixner, A. J. J. Phys. Chem. C 2008, 112, 3103-3108.

(27) Sanchot, A.; Baffou, G.; Marty, R.; Arbouet, A.; Quidant, R.; Girard, C.; Dujardin, E. ACS Nano 2012, 6, 3434-3440.

(28) Leuvering, J. H. W.; Thal, P. J. H. M.; Waart, M. V. D.; Schuurs, H. W. M. Z. Anal. Chem. Freseniu. 1980, 301, 132.

(29) Ando, J.; Fujita, K.; Smith, N. I.; Kawata, S. Nano Lett. 2011, 11, 5344-5348.

(30) Sathuluri, R. R.; Yoshikawa, H.; Shimizu, E.; Saito, M.; Tamiya, E. PloS ONE 2011, 6, e22802.

(31) Liu, S.; Tang, Z. J. Mater. Chem. 2010, 20, 24-35.

(32) Atwater, H.; Polman, A. Nat. Mater. 2010, 9, 205-213.

(33) Catchpole, K. R.; Polman, A. Appl. Phys. Lett. 2008, 93, 191113.

(34) Schaadt, D. M.; Feng, B.; Yu, E. T. Appl. Phys. Lett. 2005, 86, 063106.

(35) Girard, C.; Dujardin, E.; Li, M.; Mann, S. Phys. Rev. Lett. 2006, 97, 100801 .

(36) Sztainbuch, I. W. J. Chem. Phys. 2006, 125, 124707.

(37) Bell, S. E. J.; McCourt, M. Phys. Chem. Chem. Phys. 2009, 11, $7455-7462$.

(38) Quinten, M.; Kreibig, U.; Schönauer, D.; Genzel, L. Surf. Sci. 1985, 156, 741-750.

(39) Stranahan, S. M.; Titus, E. J.; Willets, K. A. ACS Nano 2012, 6, $1806-1813$.

(40) Lin, S.; Li, M.; Dujardin, E.; Girard, C.; Mann, S. Adv. Mater. 2005, 17, 2553-2559.

(41) Jain, P. K.; Huang, W.; El-Sayed, M. A. Nano Lett. 2007, 7, 2080-2088.

(42) Rechberger, W.; Hohenau, A.; Leitner, A.; Krenn, J. R.; Aussenegg, F. R.; Lamprecht, B. Opt. Commun. 2003, 220, 137-141.

(43) Romero, I.; Aizpurua, J.; Bryant, G. W.; García De Abajo, F. J. Opt. Express 2006, 14, 9988-9999.

(44) Lin, M. Y.; Lindsay, H. M.; Weitz, D. A.; Klein, R.; Ball, R. C.; Meakin, P. J. Phys. Condens. Mat. 1990, 2, 3093-3113.

(45) Lin, M. Y.; Lindsay, H. M.; Weitz, D. A.; Ball, R. C.; Klein, R.; Meakin, P. Nature 1989, 339, 360-362.

(46) Weitz, D. A.; Huang, J. S.; Lin, M. Y.; Sung, J. Phys. Rev. Lett. 1985, 54, 1416-1419.

(47) Dobbins, R. A.; Megaridis, C. M. Appl. Opt. 1991, 30, 47474754.

(48) An, Q.; Li, G.; Tao, C.; Li, Y.; Wu, Y.; Zhang, W. Chem. Commun. 2008, 1989-1991.
(49) Taylor, R. W.; Lee, T.-C.; Scherman, O.; Esteban, R.; Aizpurua, J.; Huang, F. M.; Baumberg, J. J.; Mahajan, S. ACS Nano 2011, 5, 3878-3887.

(50) Tao, C.-A.; An, Q.; Zhu, W.; Yang, H.; Li, W.; Lin, C.; Xu, D.; Li, G. Chem. Commun. 2011, 9867-9869.

(51) Kreibig, U.; Vollmer, M. Optical Properties of Metal Clusters; Toennies, J. P., Ed.; 2nd ed.; Springer: Berlin, Germany, 1995.

(52) Storhoff, J. J.; Lazarides, A. A.; Mucic, R. C.; Mirkin, C. A.; Letsinger, R. L.; Schatz, G. C. J. Am. Chem. Soc. 2000, 122, 46404650.

(53) Khlebtsov, N. G.; Dykman, L. A.; Krasnov, Y. M.; Mel'nikov, A. G. Colloidal J+. 2000, 62, 765-779.

(54) Quinten, M. J. Clust. Sci. 1999, 10, 319-358.

(55) Kreibig, U.; Quinten, M.; Schoenauer, D. Phys. A 1989, 157, 244-261.

(56) Lindsay, H. M.; Lin, M. Y.; Weitz, D. A.; Sheng, P.; Chen, Z.; Klein, R.; Meakin, P. Faraday Disc. Chem. Soc. 1987, 83, 153-165.

(57) Kreibig, U.; Quinten, M.; Schoenauer, D. Phys. Scr. 1986, T13, 84-92.

(58) Park, S. Y.; Lee, J.; Georganopoulou, D.; Mirkin, C. A.; Schatz, G. C. J. Phys. Chem. B 2006, 110, 12673-12681.

(59) Lazarides, A.; Schatz, G. C. J. Phys. Chem. B 2000, 104, 460467.

(60) Huang, F.; Baumberg, J. J. Nano Lett. 2010, 10, 1787-1792.

(61) Jiang, B. L.; Zhang, H.; Zhuang, J.; Yang, B.; Yang, W.; Li, T.; Sun, C. Adv. Mater. 2005, 17, 2066-2070.

(62) Sweatlock, L. A.; Maier, S. A.; Atwater, H. A.; Penninkhof, J. J.; Polman, A. Phys. Rev. B 2005, 71, 235408.

(63) Arnold, M. D.; Blaber, M. G.; Ford, M. J.; Harris, N. Opt. Express 2010, 18, 7528-7542.

(64) Atay, T.; Song, J.; Nurmikko, A. V. Nano Lett. 2004, 4, 16271631.

(65) Quinten, M.; Kreibig, U. Appl. Opt. 1993, 32, 6173-6182.

(66) Gerardy, J. M.; Ausloos, M. Phys. Rev. B 1982, 25, 4204-4229.

(67) Esteban, R.; Taylor, R. W.; Baumberg, J. J.; Aizpurua, J. Langmuir 2012, 28, 8881-8890.

(68) García de Abajo, F.; Howie, A. Phys. Rev. B 2002, 65, 115418.

(69) Johnson, P. B.; Christy, R. W. Phys. Rev. B 1972, 6, 4370-4379.

(70) Sannomiya, T.; Vörös, J.; Hafner, C. J. Comput. Theory Nanosci. 2009, 6, 749-756.

(71) Alù, A.; Engheta, N. New J. Phys. 2010, 12, 013015.

(72) Markel, V.; Sarychev, A. Phys. Rev. B 2007, 75, 085426.

(73) Barrow, S. J.; Funston, A. M.; Gómez, D. E.; Davis, T. J.; Mulvaney, P. Nano Lett. 2011, 11, 4180-4187.

(74) Rüting, F. Phys. Rev. B 2011, 83, 115447.

(75) Ortiz, G. P.; Mochán, W. L. Phys. Rev. B 2003, 67, 184204.

(76) Udagedara, I. B.; Rukhlenko, I. D.; Premaratne, M. Opt. Express 2011, 19, 19973.

(77) Conforti, M.; Guasoni, M. J. Opt. Soc. Am. B 2010, 27, 15761582 .

(78) Quinten, M. Appl. Phys. B Lasers O. 2001, 73, 245-255.

(79) Markel, V.; Shalaev, V.; Zhang, P.; Huynh, W.; Tay, L.; Haslett, T.; Moskovits, M. Phys. Rev. B 1999, 59, 10903-10909.

(80) Stockman, M. I.; Shalaev, V. M.; Moskovits, M.; Botet, R.; George, T. F. Phys. Rev. B 1992, 46, 2821-2830.

(81) Imura, K.; Okamoto, H.; Hossain, M. K.; Kitajima, M. Nano Lett. 2006, 6, 2173-2176.

(82) Brandl, D. W.; Mirin, N.; Nordlander, P. J. Phys. Chem. B 2006, $110,12302-12310$.

(83) Hao, E.; Schatz, G. C. J. Chem. Phys. 2004, 120, 357-366.

(84) Wang, Z. B.; Luk'yanchuk, B. S.; Guo, W.; Edwardson, S. P.; Whitehead, D. J.; Li, L.; Liu, Z.; Watkins, K. G. J. Chem. Phys. 2008, 128, 094705.

(85) Bonell, F.; Sanchot, A.; Dujardin, E.; Péchou, R.; Girard, C.; Li, M.; Mann, S. J. Chem. Phys. 2009, 130, 034702.

(86) Taylor, R.; Esteban, R.; Aizpurua, J.; Baumberg, J. J.; Mahajan, $\mathrm{S}$., in preparation.

(87) Barrera, R. G.; Monsivais, G.; Mochán, W. L. Phys. Rev. B 1988, $38,5371-5379$. 
(88) Rojas, R.; Claro, F. Phys. Rev. B 1986, 34, 3730-3736.

(89) Markel, V.; Shalaev, V.; Stechel, E.; Kim, W.; Armstrong, R. Phys. Rev. B 1996, 53, 2425-2436.

(90) Hu, M.; Novo, C.; Funston, A.; Wang, H.; Staleva, H.; Zou, S.; Mulvaney, P.; Xia, Y.; Hartland, G. V. J. Mater. Chem. 2008, 18, 19491960.

(91) Taking $\omega_{\mathrm{p}}=9.06 \mathrm{eV}$ and $\varepsilon_{\infty}=9$.

(92) Wark, A. W.; Stokes, R. J.; Darby, S. B.; Smith, W. E.; Graham, D. J. Phys. Chem. C 2010, 114, 18115-18120.

(93) Khan, I.; Cunningham, D.; Littleford, R. E.; Graham, D.; Smith, W. E.; McComb, D. W. Anal. Chem. 2006, 78, 224-230.

(94) Noguez, C.; Barrera, R. G. Phys. A 1994, 211, 399-410.

(95) Noguez, C.; Barrera, R. G. Phys. Rev. B 1998, 57, 302-313. 